\title{
GHZ RANGE NANOSCALED ALN CONTOUR-MODE RESONANT SENSORS (CMR-S) WITH SELF-SUSTAINED CMOS OSCILLATOR
}

\author{
M. Rinaldi ${ }^{*}$, C. Zuniga, C. Zuo and G. Piazza \\ University of Pennsylvania, Philadelphia, USA
}

\begin{abstract}
This paper reports on the design and experimental verification of a new class of nanoscaled AIN Contour-Mode Resonant Sensors (CMR-S) for the detection of volatile organic chemicals (VOC) operating at frequencies above $1 \mathrm{GHz}$ and connected to a chip-based CMOS oscillator circuit for direct frequency read-out. This work shows that by scaling the CMR-S to $250 \mathrm{~nm}$ in thickness and by operating at high frequencies $(1 \mathrm{GHz})$ a limit of detection of $\sim 35 \mathrm{zg} / \mu \mathrm{m}^{2}$ and a fast response time $(<1 \mathrm{~ms})$ can be attained. In addition, the capability to detect concentrations of volatile organic compounds such as 2,6 dinitroluene (DNT) as low as $1.5 \mathrm{ppb}\left(4.7 \mathrm{ag} / \mu^{2}\right)$ is experimentally verified.
\end{abstract}

\section{INTRODUCTION}

In recent years the demand of highly miniaturized sensor arrays capable of selectively detecting extremely small concentrations (part per trillion, ppt) of multiple gaseous analytes has steadily grown. The necessity to detect such small concentrations requires reliably measuring extremely small variations in the sensor output signal. In this perspective, gravimetric sensors, such as Quartz Crystal Microbalances (QCMs), have a significant advantage over other sensor technologies (conductance-based sensors, Chem-FET, or optical sensors) since they use frequency as the output variable, which is one of the physical quantities that can be monitored with the highest accuracy [1].

Nevertheless, smart, compact, portable and low cost electronic noses for multiple gas detection cannot be implemented with bulky and unintegrable QCMs. Even if QCMs have been successfully employed as gravimetric sensors thanks to their extremely high quality factors, which permit to obtain limits of mass detection in the order of few nanograms, their relatively large volume and their inability to be directly integrated on silicon render them unattractive for the fabrication of sensor arrays composed of a large number of mechanical elements.

In response to this growing demand for miniaturization, the use of micro and nano electromechanical (MEMS/NEMS) resonators for sensing applications has been extensively explored. The scaling of the device dimensions enables the fabrication of ultra-sensitive nanomechanical gravimetric sensors that can be arranged in single-chip arrays capable of selectively detecting extremely small concentrations (ppb-ppt) of multiple gaseous analytes.

Different MEMS/NEMS resonator technologies based on electrostatic or piezoelectric transduction [2,3] or piezoresistive displacement transduction [4] have been proposed. In particular, NEMS resonators have been exploited as transducers for the realization of extremely sensitive gravimetric sensors. Subattogram mass resolution has been demonstrated in NEMS cantilevers [4] thanks to the minuscule mass and the relatively high quality factor $(Q)$. Nevertheless the greatly reduced dimensions of these devices render their transduction extremely difficult and require the use of cumbersome, complex and power inefficient read-out techniques. In this perspective, the employment of large surface area devices with nanoscaled thickness (such as flexural membranes [5] or laterally vibrating contour-mode resonators [6]), instead of beams, is advantageous in the making of gravimetric sensors since it enables the fabrication of extremely low mass devices with power efficient transduction and readout.

In addition, the scaling of micro/nanoelectromechanical resonators to $\mathrm{GHz}$ frequencies, hence higher sensitivity to mass loading, has been a significant challenge because the device transduction tends to become inefficient and its performance is generally accompanied by a reduction in quality factor, $Q$. These issues have prevented connecting high frequency nanoelectromechanical resonators to self-sustaining oscillators, therefore rendering real-time VOC sensing measurements impossible.

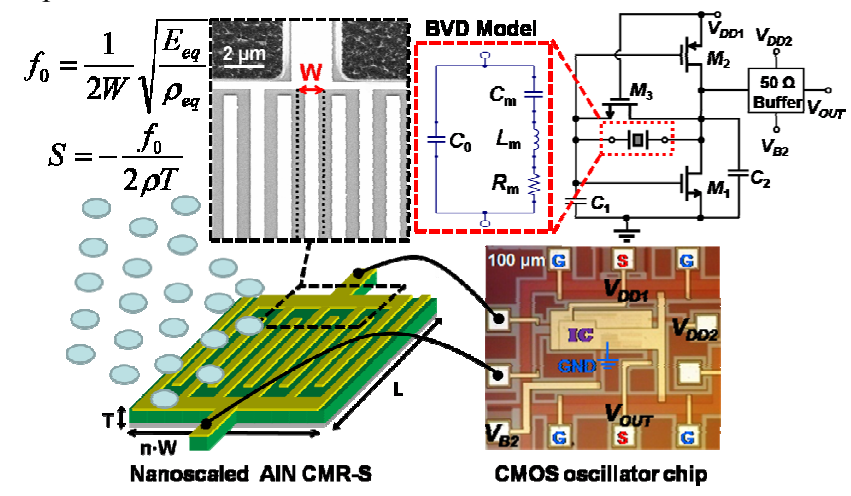

Figure 1: Schematic representation of the fabricated nanoscaled AlN CMR-S connected to a self-sustained CMOS oscillator circuit. $A$ number, $n$, of subresonators (width, $W$ ) are mechanically coupled in order to excite a higher mode of vibration in the AlN plate $\left(f_{0}\right.$ is set by $W$ ). The butterworth van dyke (BVD) model is used to describe the resonator behavior.

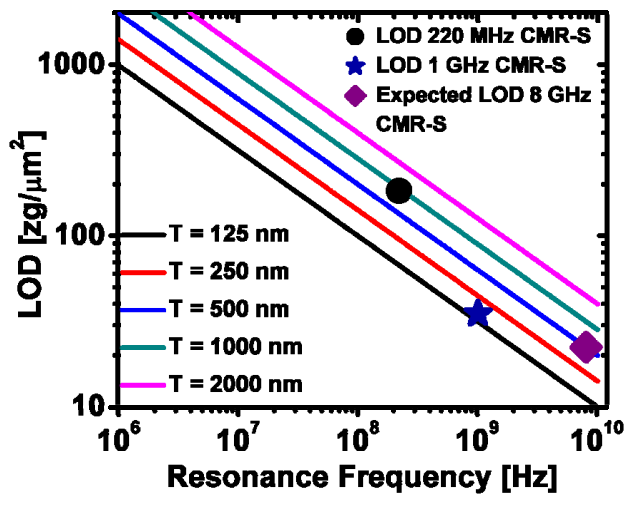

Figure 2: Limit of detection as function of the CMR-S resonance frequency and thickness. The device surface area is assumed to be constant over frequency and the input power is assumed to be the device critical power (maximum power before non-linear behavior). The measured data points follow the predicted trend.

In this work, fundamental transduction problems in NEMS resonators were solved by using on-chip piezoelectric actuation and sensing of an ultra-thin $(250 \mathrm{~nm})$ AlN plate (equivalent to a membrane) formed of AIN nanostructures mechanically coupled 
and forced to simultaneously vibrate at the same frequency $(\mathrm{GHz}$ range) in the contour-extensional mode (Fig. 1). A low frequency ( $220 \mathrm{MHz})$ ultra-thin-film AlN CMR-S for VOC detection was previously demonstrated by our group [6]. In this work, the sensor performance in terms of LOD (Fig.2), or measurement speed for a given LOD (Fig.3), were improved by scaling the device to higher frequencies of operation.

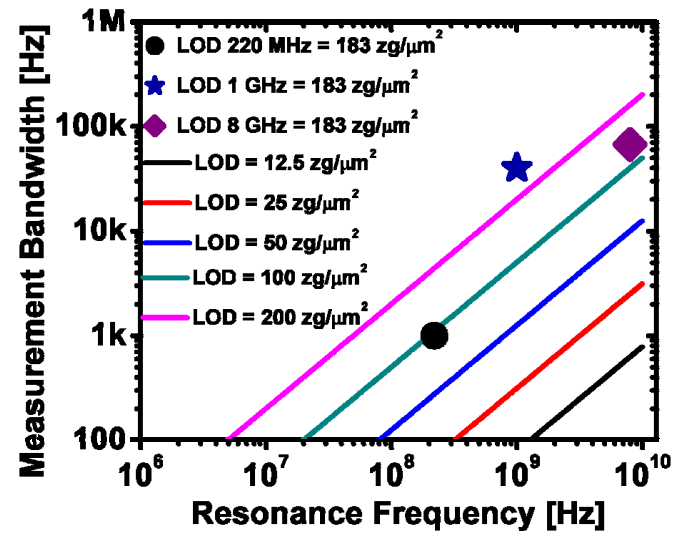

Figure 3: Measurement bandwidth (i.e. speed) as a function of the $C M R-S$ resonance frequency for a given LOD. Note that the experimental data are in line with theoretical predictions.

Ultra-thin-film (250-500 nm) AlN CMR-S operating at frequencies as high as $8 \mathrm{GHz}$ with figure of merit $\left(\mathrm{FOM}=k_{t}^{2} \cdot Q\right.$, product of the electromechanical coupling and resonator $Q$ ) in excess of 10 and impedances below $100 \Omega$ were experimentally demonstrated. High values of sensitivity to mass loading $(\sim 290$ $\mathrm{KHz} \cdot \mu \mathrm{m}^{2} / \mathrm{fg}$ at $\left.1 \mathrm{GHz}\right)$ were attained thanks to the nanoscaled thickness of the AlN resonators and their high frequency of operation. Because of the large value of the resonator FOM, direct wire-bonding of nano-CMR-S, operating up to $1 \mathrm{GHz}$, to a Pierce oscillator fabricated in the ON-Semiconductor $0.5 \mu \mathrm{m}$ CMOS process was made possible (Fig. 1). The insertion of the $1 \mathrm{GHz}$ CMR-S in the oscillator loop permitted to measure a minimum Allan Variance of $10 \mathrm{~Hz}$, which translates in an ultimate LOD of $35 \mathrm{zg} / \mu^{2}{ }^{2}$ with a measurement bandwidth of solely $1 \mathrm{~ms}$.

These unprecedented results have not been demonstrated by any other micro/nanoscale resonant sensor $[5,7,8]$. By operating the CMR-S technology at high frequency, which is associated with an increase in CMR-S mass sensitivity and power handling (i.e. better phase noise performance) [9], a lower LOD and a higher measurement speed for a given LOD (Fig. 2-3) were attained.

In order to test the sensing properties of one of the fabricated devices, its top metal electrode was made out of gold, given its known ability to adsorb organic molecules in vapor phase and its capability to be eventually functionalized with thiol terminated self assembled monolayers for selective affinity enhancement towards specific analytes [10]. The device was exposed to different concentrations of 2,6 dinitroluene (DNT) and, despite the lack of an affinity enhancing functionalization layer, the ability to detect concentrations as low as $1.5 \mathrm{ppb}\left(4.7 \mathrm{ag} / \mu^{2}\right)$ was demonstrated. This constitutes a $\sim 10 \mathrm{X}$ improvement in mass per unit area detection capability compared to the one previously demonstrated by the same group with lower frequency nano-CMR-S [6].

\section{DESIGN}

A CMR is composed of an AlN film sandwiched between two patterned metal electrodes (Fig. 1). When an AC votlage is applied across the thickness $(T)$ of the device a contour-extensional mode of vibration is excited thorough the equivalent $d_{31}$ piezoelectric coefficient of AlN. Given the equivalent mass density, $\rho_{e q}$, and Young's modulus, $E_{e q}$, of the material stack (AlN and metal electrodes) that forms the resonator, the center frequency, $f_{0}$, of this laterally vibrating mechanical structure, is set by the period, $W$, of the metal electrode patterned on the AlN plate and can be approximately expressed as in Eq. (1).

$$
f_{0}=\frac{1}{2 W} \sqrt{\frac{E_{e q}}{\rho_{e q}}}
$$

The other two geometrical dimensions, thickness, $T$, and length, $L$, set the equivalent electrical impedance of the resonator [3] and can be designed independently of the desired resonance frequency.

The sensitivity to mass per unit area of a CMR-S loaded on its top surface can be expressed as in Eq. (2) [11, 12].

$$
S_{C M R-S}=-\frac{f_{0}}{2 \rho_{e q} T}
$$

It is worth nothing that the resonance frequency, $f_{0}$, and the thickness, $T$, of the CMR-S can be set independently so as to achieve the desired value of sensitivity. In particular, the device sensitivity can be improved by scaling the thickness, $T$, of the AlN film and by increasing the device resonance frequency, $f_{0}$.

Nevertheless, the mass per unit area sensitivity of the device cannot be considered the only important parameter for the design of a high performance gas sensor. In fact, the limit of detection, $L O D$, of the sensor (smallest amount of adsorbed mass per unit area that can be resolved) needs to be taken in to account:

$$
L O D=\frac{\Delta f_{\min }}{S_{C M R-S}}
$$

where $\Delta f_{\text {min }}$ indicates the minimum frequency shift detectable by the sensor read-out. When the resonant sensor is connected to a self-sustained oscillator loop for direct frequency readout, the minimum detectable frequency shift, $\Delta f_{\min }$, is determined by the phase noise of the oscillator. Therefore, the performance of the sensor can be improved by reducing the thickness, $T$, of the AIN layer and by increasing the device resonance frequency, $f_{0}$ (i.e. improving the device sensitivity) up to the limit at which good phase noise is preserved.

According to these guidelines, the CMR-S of this work were scaled both in the vertical $(T)$ and lateral $(W)$ directions and a lateral field excitation scheme (LFE) was employed to excite a higher order contour-extensional mode of vibration in the nanoscaled AIN structures. Despite the volume reduction and the increase in the resonance frequency, high $Q(\sim 700$ in air at $8 \mathrm{GHz})$ and high $k_{t}^{2}(>1.5 \%)$ were still attained. Such high values of the device figure of merit, $k_{t}^{2} \cdot Q$, enable the direct connection of the CMR-S to a compact and low power self-sustained oscillator circuit for direct frequency read-out. In fact, the primary power loss in this oscillator circuit is due to the motional resistance, $R_{m}$, of the resonator [13], whose value is inversely proportional to the device figure of merit, $k_{t}^{2} \cdot Q[14]$.

The oscillator circuit topology used as read-out for the CMR$\mathrm{S}$ of this work is described in [13]. Briefly, the circuit (Fig. 1) consists of a Pierce oscillator implemented by means of a CMOS inverter biased in its active region. Transistors M1 and M2 form the CMOS inverter while transistor M3 acts as a large resistor to provide biasing in the active region of M1 and M2.

\section{GHz nano-CMR-S design}

In order to improve the device sensitivity to mass loading according to equation 2 the frequency setting dimension, $W$, was 
set to $4 \mu \mathrm{m}$ (frequency of operation of $\sim 1 \mathrm{GHz}$ ) and the thickness of the AlN film was scaled to $250 \mathrm{~nm}$. The very thin AlN film (composing the body of the resonator) was deposited on top of a floating bottom Platinum (Pt) electrode which acts to confine the electric field across the thickness of the device and provides for a high $k_{t}^{2}(1.7 \%$ in this work).

\section{$8 \mathrm{GHz}$ nano-CMR-S design}

The capability to fabricate nanoscaled CMR-S operating in the Super High Frequency (SHF) band $(>3 \mathrm{GHz}$ ) has been previously demonstrated by our group [15]. Nevertheless, the motional impedance, $R_{m}$, of that device was considerably high $(\sim 1$ $\mathrm{K} \Omega$ ) and not suitable for the implementation of compact and low power self-sustained oscillators for direct sensor frequency readout. In this work a substantial step forward towards the implementations of such sensor system has been made by designing a nano-CMR-S operating at $8 \mathrm{GHz}$ with a value of motional resistance, $R_{m}$, of $\sim 70 \Omega$.

To operate at $\sim 8 \mathrm{GHz}$, the frequency setting dimension, $W$, was scaled into the nanodomain $(\mathrm{W}=600 \mathrm{~nm})$ and no bottom electrode was employed in order to maintain extremely high quality AlN. The nano AIN film was directly deposited on top of a Si substrate and its thickness set to $500 \mathrm{~nm}$ in order to maximize the electromechanical coupling, $k_{t}^{2}$, given a resonance frequency of $8 \mathrm{GHz}[16]$.

\section{EXPERIMENTAL RESULTS}

A 4 mask fabrication process, analogue to the one reported in [12], was employed to fabricate the devices presented in this work.

The electrical response of the fabricated ultra-thin-film AlN CMR-S was characterized in ambient conditions in an RF probe station and the admittance curve measured by an Agilent ${ }^{\mathbb{B}}$ N5230A Network Analyzer after performing a short-open-load (SOL) calibration on a reference substrate.

Both the experimental and fitted admittance curves of the fabricated high frequency CMR devices are shown in Figures 4 and 5 .

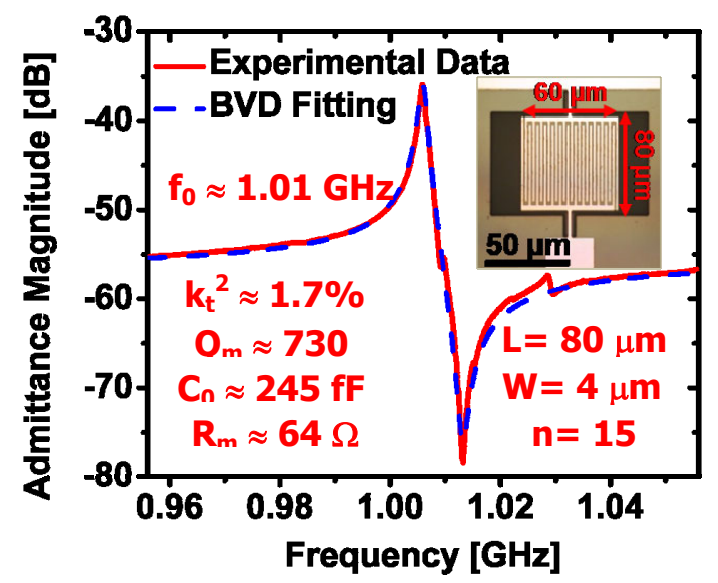

Figure 4: Experimental and BVD fitted admittance curves of the fabricated $1 \mathrm{GHz}$ nano CMR-S (70 $\mathrm{nm}$ thick top Al electrode).

Despite the device volume reduction, $k_{t}^{2} \cdot Q$ products as high as 12.4 and 5.6 were attained at $1 \mathrm{GHz}$ and $8 \mathrm{GHz}$, respectively.

These characterstics enabled the demonstration of the first $\mathrm{GHz}$ range oscillator based on nanoscaled AIN CMR-S. The 1 $\mathrm{GHz}$ device was directly wire-bonded to the oscillator circuit chip fabricated in the ON Semiconductor $0.5 \mu \mathrm{m}$ CMOS process. In order to estimate the noise limited minimum frequency shift detectable by the sensor read-out $\left(\Delta f_{\text {min }}\right)$, the short term frequency stability of the oscillator output signal was characterized by its phase-noise derived Allan variance, $\sigma_{y}(\tau)$. An equivalent value of Allan variance of $\sim 10 \mathrm{~Hz}$ was derived for a measurement time of 1 ms (Fig.6). Therefore, considering a mass sensitivity of $\sim 290$ $\mathrm{KHz} \cdot \mu \mathrm{m}^{2} / \mathrm{fg}$ (extracted according to [12]) and a minimum detectable frequency shift of $10 \mathrm{~Hz}$, a LOD of mass per unit area of $\sim 35 \mathrm{zg} / \mu^{2}$ can be estimated. The experimentally derived values of LOD and measurement bandwidth confirm that resonant sensor performances are significantly improved by scaling the CMR-S device thickness and moving from megahertz to gigahertz frequencies.

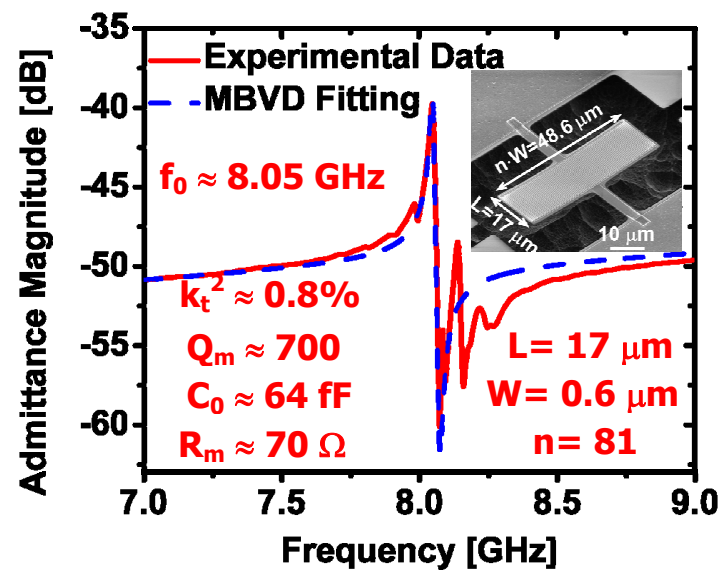

Figure 5: Experimental and MBVD fitted admittance curves of the fabricated $8 \mathrm{GHz}$ nano $C M R-S$.

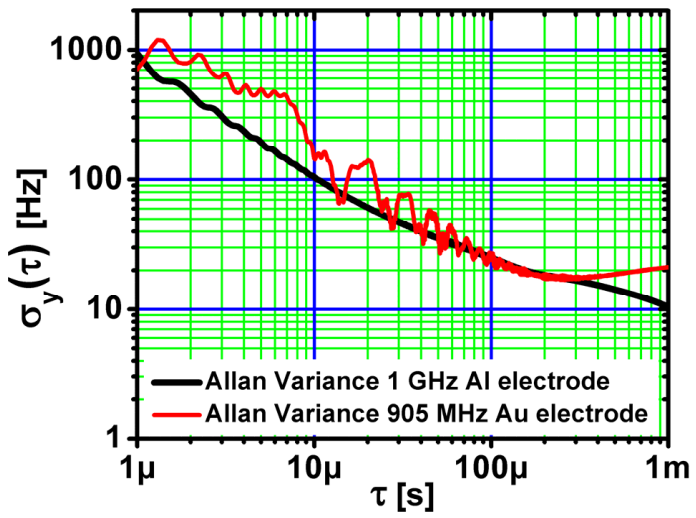

Figure 6: Allan variances for different values of integration time, $\tau$, calculated from the measured phase noise of the fabricated 1 $\mathrm{GHz}$ oscillator (using an Agilent $\mathrm{R}$ 8562EC spectrum analyzer) and of the $905 \mathrm{MHz}$ oscillator (using an Agilent $\AA$ E5052B Signal Source Analyzer).

In order to test the sensing properties of one of the fabricated devices, its top metal electrode was made out of gold $(40 \mathrm{~nm})$, given its known ability to adsorb organic molecules in vapor phase [10]. The geometry of the device is the same of the one reported in Fig. 4 but its measured resonance frequency is lower $(\sim 905 \mathrm{MHz})$ because of the difference in thickness and mass density between the gold and the aluminum electrodes.

Both the resonator die and the CMOS chip were attached to a custom designed PCB, which served as the lid for the testing chamber [12]. The phase-noise of the gold electrode nano-CMR-S oscillator was measured with an Agilent ${ }^{\circledR}$ E5052B Signal Source Analyzer (Fig. 6-7) and found to be comparable to the one observed in the lower frequency nano-CMR-S oscillator (Fig. 7). 
The sensor was exposed to DNT vapor (generated by bubbling method [12]) and the frequency shift due to the adsorption of the analyte on the $\mathrm{Au}$ electrode (without any functionalization layer) was monitored with the spectrum analyzer functionality of an Agilent ${ }^{\circledR}$ E5052B Signal Source Analyzer (Fig. 8). The sensor was exposed to different concentrations of DNT ranging between $0.2 \%$ and $1 \%$ (Table 1 ) of the saturated vapor pressure and a minimum frequency shift of $\sim 1 \mathrm{KHz}$ was detected in correspondence of the $0.2 \%$ of the DNT saturated vapor pressure (Table 1). Considering that the DNT saturated vapor pressure at room temperature is equal to $5.67 \cdot 10^{-4}$ Torr [17], a concentration of DNT as low as $1.5 \mathrm{ppb}$ was detected by the fabricated nanoenabled CMR-S prototype. Given the device sensitivity of $\sim 210 \mathrm{KHz} \cdot \mu \mathrm{m}^{2} / \mathrm{fg}$ (extracted according to [12]) and the minimum frequency shift of $\sim 1 \mathrm{KHz}$ a LOD of mass per unit area of $4.7 \mathrm{ag} / \mu \mathrm{m}^{2}$ was experimentally verified.

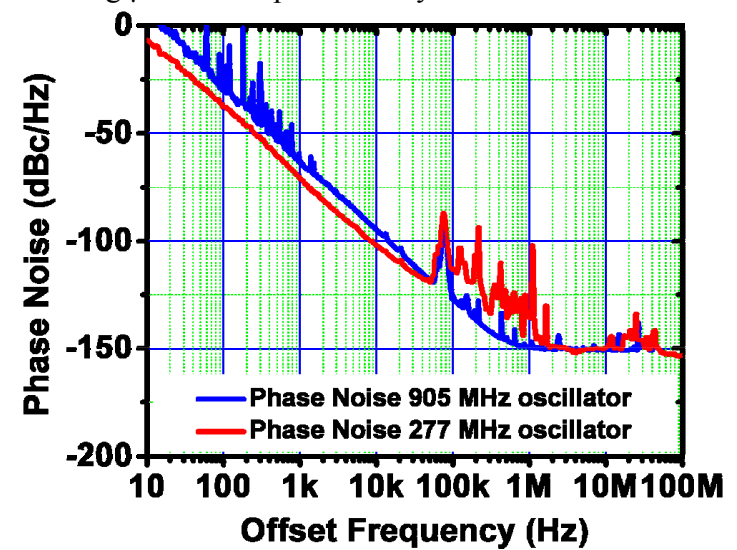

Figure 7: Measured phase noises of a $905 \mathrm{MHz}$ and $277 \mathrm{MHz}$ oscillators based on nano-CMR-S. A phase noise of $71.3 \mathrm{dBc} / \mathrm{Hz}$ at $1 \mathrm{KHz}$ frequency offset from the carrier is recorded for the $905 \mathrm{MHz}$ oscillator.

Table 1: Sensor response to different concentrations of DNT.

\begin{tabular}{|c|c|c|}
$\begin{array}{c}\text { DNT Vapor } \\
\text { Pressure } \\
\text { Percentage (\%) }\end{array}$ & $\begin{array}{c}\text { DNT } \\
\text { Concentration } \\
(\mathbf{p p b})\end{array}$ & $\begin{array}{c}\text { Frequency Shift } \\
(\mathbf{K H z})\end{array}$ \\
\hline 1 & 7.46 & 6.5 \\
\hline 0.5 & 3.73 & 3.4 \\
\hline 0.25 & 1.86 & 1.7 \\
\hline 0.2 & 1.49 & 1 \\
\hline
\end{tabular}

Despite the lack of an affinity enhancing functionalization layer an extremely low concentration of DNT (1.5 ppb) was detected. This LOD of DNT concentration is expected to reduce by about $8 \mathrm{X}(\sim 190 \mathrm{ppt})$ when a nano-bio coating layer such as the one reported in [6] is employed.

\section{CONCLUSION}

In this paper, the design and the experimental verification of nanoscale $\mathrm{GHz}$ range gravimetric sensors based on ultra-thin film AlN CMR-S connected to a chip-based self-sustaining oscillator loop for direct frequency read-out was demonstrated. Devices operating up to $8 \mathrm{GHz}$ with values of FOM within 5.6 and 12.4 were experimentally demonstrated. An ultimate LOD of mass per unit area of $\sim 35 \mathrm{zg} / \mu \mathrm{m}^{2}$ for a $1 \mathrm{GHz}$ sensor was estimated thanks to the high frequency stability of the oscillator signal (minimum Allan variance of $10 \mathrm{~Hz}$ for $1 \mathrm{~ms}$ bandwidth). Concentrations of DNT as low as $1.5 \mathrm{ppb}$ were experimentally detected and correspond to a value of adsorbed mass per unit area of $4.7 \mathrm{ag} / \mu \mathrm{m}^{2}$. These results constitute a $\sim 10 \mathrm{X}$ improvement in mass per unit area limit of detection compared to previously demonstrated low frequency nano-CMR-S and confirm that resonant sensor performances are significantly enhanced by scaling the CMR-S device thickness and moving from megahertz to gigahertz frequencies.

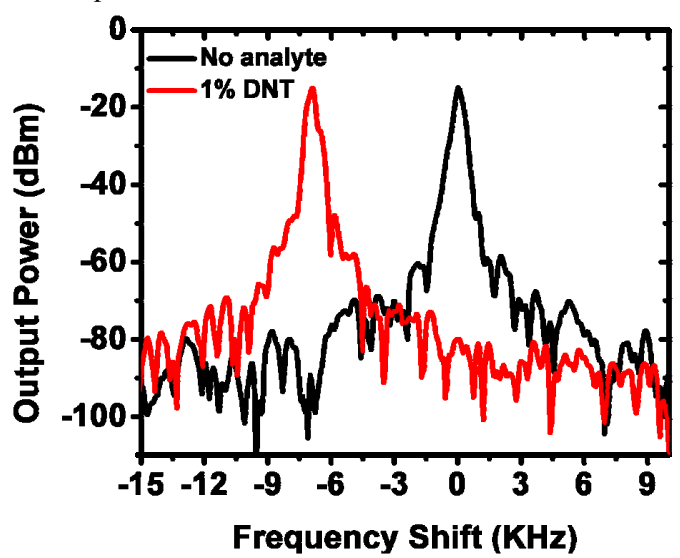

Figure 8: Shift of the oscillator spectrum corresponding to exposure to $1 \%$ DNT saturated vapor pressure. The detection of smaller shifts, corresponding to lower concentrations, was attained by averaging over various measurements.

\section{REFERENCES}

[1] J. R. Vig, L. Filler, Y. Kim, J. Microelectromech. Syst., vol. 5, no. 2, pp. 131-137, 1996.

[2] K. Wang, A.-C. Wong and C. T.-C. Nguyen, J. Microelectromech. Syst., vol. 9, no. 3, pp. 347-360, 2000.

[3] G. Piazza, P.J. Stephanou, A.P. Pisano, J. Microelectromech. Syst., vol. 15, no.6, pp. 1406-1418, 2006.

[4] M. Li, H. X. Tang, M.L. Roukes, Nature Nanotechnology, vol. 2, pp. 114-120, 2007.

[5] H. J. Lee, K. K. Park, P. Cristman, Ö. Oralkan, M. Kupnik, and B. T. Khuri-Yakub, Proc. IEEE MEMS 2009, Jan. 2009, pp. 761-764.

[6] M. Rinaldi, B. Duick, C. Zuniga, C. Zuo and G. Piazza, Proc. IEEE MEMS 2010, Jan. 2010, pp. 132-135.

[7] Feng, X.L., White, C.J., Hajimiri, A. and Roukes, M.L, Nature Nanotechnology, 3, 342-346, 2008.

[8] M. Penza et al, IEEE Trans. Electron Device, 55, 1237, 2008.

[9] C. Zuo, M. Rinaldi and G. Piazza, Proc. IEEE International Ultrasonics Symposium 2009, Sep. 2009, pp. 1187-1190.

[10] T. M. Herne and M. J. Tarlov, J. Am. Chem. Soc., 119, pp. 89168920, 1997.

[11] C. Zuniga, M. Rinaldi, S. M. Khamis, A. T. Johnson, and G. Piazza, Applied Physics Letters, vol. 94, 223122, 2009.

[12] M. Rinaldi, C. Zuniga, and G. Piazza, Poc. IEEE International Ultrasonics Symposium, Sept. 2009, pp. 714-717.

[13] C. Zuo, J. Van der Spiegel, and G. Piazza. Proceedings IEEE EFTFIFCS 2009, April 2009, pp. 381-384.

[14] K.M. Lakin, Proc. IEEE MTT-S Int. Microwave Symp. Dig., June 1992, pp. 149-152.

[15] M. Rinaldi, C. Zuniga, and G. Piazza, Proc. IEEE MEMS 2009, Jan. 2009, pp. 916-919.

[16] M. Benetti, D. Cannata, F. Di Pietroantonio, and E. Verona, Poc. IEEE IUS 2007, Sept. 2007, pp. 1673-1676.

[17] Regulatory Determinations Support Document for Selected Contaminants from the Second Drinking Water Contaminant Candidate List (CCL 2), United States Environmental Protection Agency, Chapter 7, June 2008.

\section{CONTACT}

*M. Rinaldi, tel: +1-215-573-3276; rinaldim@seas.upenn.edu 